\title{
Revealing the mechanism of lymphoid and myeloid cell differentiation and transdifferentiation through landscape quantification
}

\author{
Chenyong Zhang ${ }^{1}$ and Chunhe $\mathrm{Li} \odot^{1,2, *}$ \\ ${ }^{1}$ Shanghai Center for Mathematical Sciences, School of Mathematical Sciences, Fudan University, Shanghai 200433, China \\ ${ }^{2}$ Institute of Science and Technology for Brain-Inspired Intelligence, Fudan University, Shanghai, 200433, China
}

(Received 22 September 2020; accepted 9 February 2021; published 26 February 2021)

\begin{abstract}
The hematopoietic stem cells (HSCs) can differentiate to myeloid and lymphoid lineages, which lead to more specific differentiated cells. This cell fate decision process is governed by an underlying gene regulatory network. However, its underlying mechanism remains to be fully clarified. In this work, based on a gene regulatory network of HSC differentiation, we built a dynamical model and developed an optimization approach to infer model parameters by fitting experimental data quantitatively. We mapped out the potential landscape of HSC differentiation and identified multistable cell states observed in experiments, including multipotent progenitor, B-cell, and macrophage. Interestingly, we also identified one new intermediate cell state on the landscape, which plays a critical role on the cellular transdifferentiation. By calculating the transition action among different attractor states, we quantified kinetic transition paths for differentiation and transdifferentiation between different cell states and identified key genes and regulations during these transition processes. Our work provides a general approach to construct a gene regulatory network model based on experimental data and new insights into the roles of intermediate states on the transdifferentiation process in HSC development.
\end{abstract}

DOI: 10.1103/PhysRevResearch.3.013186

\section{INTRODUCTION}

Hematopoietic stem cells (HSCs), which are capable of self-renewal and differentiating into almost all blood cell lineages, served as a paradigm for understanding stem cell development [1,2]. Mounting researches have been focused on this topic [1-6]. Mathematical modeling approaches are gradually introduced to study gene regulatory networks and capture temporal evolving characteristics of the system [7]. For example, computational models are used to reconstruct the gene regulatory network of HSC systems [8]. Also, Boolean models of gene regulatory networks have been used to study the differentiation mechanisms and identify key interactions in cell fate commitment $[9,10]$.

However, the stochastic nature of HSC differentiation systems was not well elucidated in these previous studies [8-10]. The characteristics of a kinetic system such as global stability of different cell states and dynamical transitions between different cell states under intrinsic and external noise remain challenging to quantify [11-13]. To understand the process of cell fate decisions, Waddington proposed a landscape concept as a metaphor to explain the development and differentiation of cells [14]. In Waddington landscape theory, the developmental process is viewed as a ball rolling from one basin representing stem cell states to the basins representing differentiated cell states. The barrier of different basins

\footnotetext{
*chunheli@fudan.edu.cn

Published by the American Physical Society under the terms of the Creative Commons Attribution 4.0 International license. Further distribution of this work must maintain attribution to the author(s) and the published article's title, journal citation, and DOI.
}

therefore can be calculated to quantify the transition feasibility from one cell state to another one. Such framework has been used to depict the differentiation and transdifferentiation of cells, and different approaches have been developed to construct the Waddington landscapes in biological systems $[13,15-21]$.

In gene network models, parameters such as the activation or inhibition strengths of regulations are important to characterize the system. However, the values of these parameters are rarely available in literatures and often hard to measure in experiments. So, how to infer the parameter values is a general issue in gene network modeling. There have been studies performing parameter inference based on time series data [22-25]. However, for gene regulatory network models, reasonable time series data are not available in many cases, which warrants the development of new experimental techniques. Therefore, inferring parameters of gene network models from static expression data (or steady-state data) is crucial to the modeling approach, which is still a great challenge [23]. Random parameter search is normally used in related problems due to its simplicity and effectiveness, though it may require huge cost of time and fail to obtain steady states that most agree with the experimental data. Gradient descent algorithms have been flourishing since the booming of machine learning and have been applied in certain fields of biology [26]. Efforts have also been made to use gradient descent algorithms for parameter inference and landscape construction from single-cell data [27]. Here, we aim to develop an optimization approach by combining the gradient descent algorithm and random parameter search to infer model parameters from experimental data. By applying our parameter inference approach to a twodimensional gene network case, we validated the efficacy of our approach. 


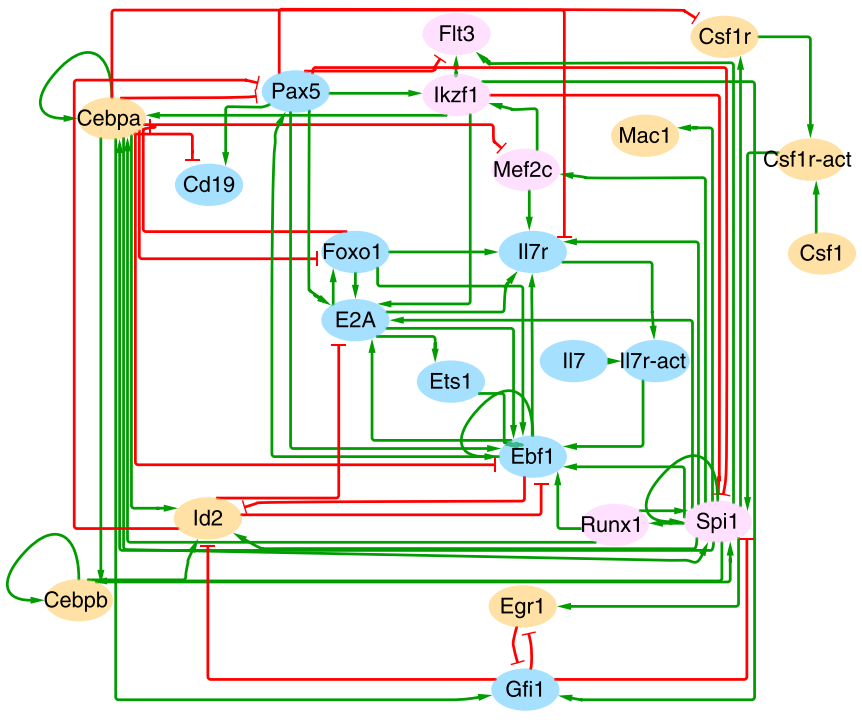

FIG. 1. Gene regulatory network of the HSC differentiation and transdifferentiation process, including 23 nodes and 71 regulations (52 activations and 19 inhibitions). Nodes represent genes (except for Csf1r-act and Il7r-act, which represent the activated forms of the receptor), and Edges represent regulations. Orange nodes denote marker genes for macrophages (Mac), blue nodes denote marker genes for B-cells, and purple nodes denote marker genes for multipotent progenitors (MP). Green and red edges represent activations and inhibitions, respectively.

To study the mechanisms of cell fate decisions in HSCs, which can differentiate to B-cells and macrophages (Mac), we built a dynamical model of HSC differentiation based on the gene regulatory network structure of HSC differentiation suggested in previous work [8]. We further applied our parameter inference approach to the HSC model and determined the model parameters. By a self-consistent mean filed approximation [18,28,29], we quantified the potential landscape of HSC differentiation. Our landscape model successfully replicates three stable cell states representing multipotent progenitor (MP), B-cell, and Mac observed in experiments. Interestingly, we also identified another intermediate state between B-cell and Mac. The intermediate state, though not yet observed in experiments, was found to play a crucial role during the transdifferentiation process between B-cell and Mac from our modeling. By calculating the minimum action paths (MAPs), we studied the transition action among different cell states, which quantified corresponding transition rate. By global sensitivity analysis, we identified key regulations during the HSC differentiation and transdifferentiation process.

\section{RESULTS}

\section{A. Mathematical modeling of HSC development}

To build the gene regulatory network model of HSC differentiation, we need the underlying network structure. Here, we used the gene network for HSC differentiation from recent work by Collombet et al., where they inferred the network structure by literature mining and fitting the CHIP-seq data from simulations $[8,30]$. The network structure is shown in Fig. 1. We also used quantitative experimental data to infer model parameters. The experimental data were collected from public databases $[4,8]$. In these experiments, the expression data from different stages of hematopoietic cell development were calculated through gene profiling, including a dataset for transdifferentiation from pre-B cells to macrophages [4]. The data were $\log 2$ normalized and averaged among different samples of the same type of cells [8].

The HSC differentiation is a complex cell fate decision process, and many cell types have been discovered and studied in hematopoetic hierarchy, such as multipotent progenitor (MP), common lymphoid progenitor (CLP), common myeloid progenitor (CMP), B-cell and macrophage [2,5,6,8]. Here, considering the complexity of the gene regulatory model, we focused on three major cell types including MP, B-cell and Mac, and studied three important processes including differentiation, reprogramming, and transdifferentiation. Therefore, we only used the data for these three cell types, and considered them as biological steady states in the timescale that we are interested (i.e., the timescale corresponding to the cell state transition among these three cell types). The processed gene expression data are shown in Fig. 2 (left). All the expression values were normalized to the range of 0 to 1 (relative to the highest expression of each gene among different cell states). The experimental data indicate stable cell states for MP, B-cells, and macrophages. These stable cell states should correspond to the attractor states in our dynamical models, which will be the focus of our following modeling and analysis.

The HSC network includes 23 nodes representing different genes, and 71 edges representing regulations for activations and inhibitions. Based on this gene regulatory network, we can write down the corresponding ordinary differential equations (ODEs) that mathematically describe the dynamical evolving process of the HSC development and differentiation. The Hill function is used to describe the activation or inhibition regulations between different genes, which are commonly used to mimic the cooperativity for gene regulations in gene networks [31,32]. The ODEs that govern the time evolution of gene expressions are shown as follows:

$$
\frac{d x_{i}}{d t}=f_{i}(\boldsymbol{x})=\sum_{j \in M_{i}} \frac{a_{j i} x_{j}^{n}}{s_{j i}^{n}+x_{j}^{n}}+\sum_{j \in M_{i}^{\prime}} \frac{b_{j i} s_{j i}^{n}}{s_{j i}^{n}+x_{j}^{n}}-k_{i} x_{i} .
$$

Here $x_{i}$ represents the gene expression value of the $i$ th gene among the total $N$ gene nodes, $f_{i}(\boldsymbol{x})$ represents the regulatory force on the $i$ th gene, including three terms. The first two terms are for regulations of other genes on the $i$ th gene, with parameters $a_{j i}$ and $b_{j i}$ representing the activation and inhibition constants of the regulation of the $j$ th node on the $i$ th node, respectively. $s_{j i}$ is the threshold for the Hill function, and $n$ is the Hill coefficient, which determines the degree of nonlinearity of the system $[28,33]$. The last term $\left(-k_{i} x_{i}\right)$ represents the degradation of the $i$ th gene. $M_{i}$ is the aggregation of all the genes that activate the $i$ th gene, while $M_{i}^{\prime}$ is the aggregation of all the genes that inhibit the $i$ th gene.

There are two forms of Hill functions, which have been mostly used for modeling gene regulations, i.e., the additive models and multiplicative models. Take the inhibition regulation as an example, if multiple inhibiting genes are concerned, there will be two scenarios for the assumptions. For the 
experiments

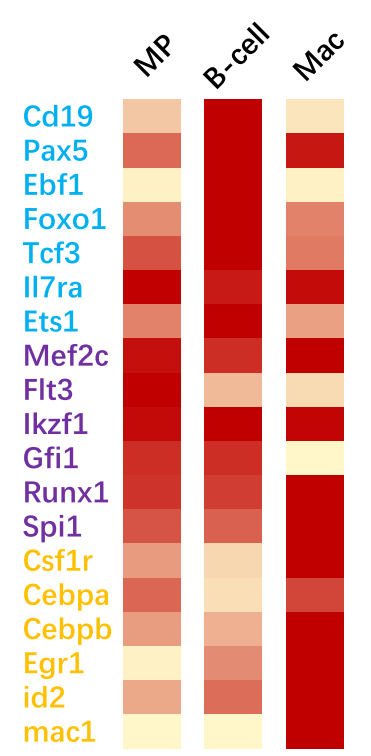

model 1

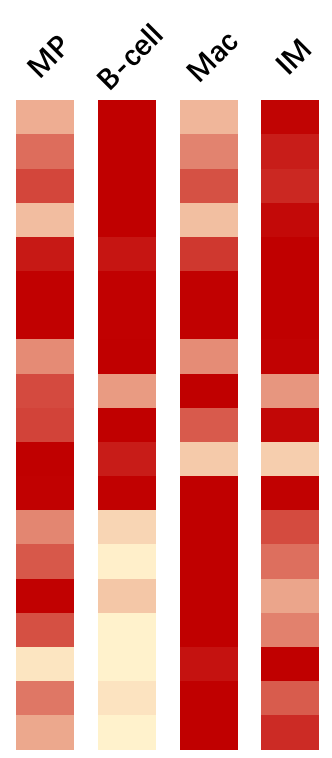

model 2

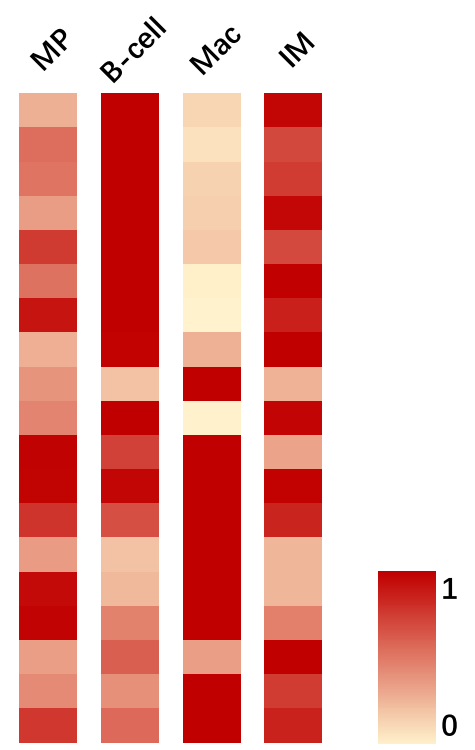

FIG. 2. Gene expression values in MP, B-cell, and Mac from experimental data (experiments) and simulation results (models 1 and 2). Expression values are normalized to range 0 to 1 (relative to the highest expression value). MP represents multipotent progenitors, Mac represents macrophages, and IM represents intermediate state. Models 1 and 2 represent the results of optimization algorithm, starting from the best initial parameter values and the second best initial parameter values (from random parameter search), respectively

multiplicative model, any one of the activated inhibitors can lead to the target gene inhibited. For the additive model, suppose we have two inhibition terms with maximal synthesis rate $\mathrm{b} 1$ and $\mathrm{b} 2$, the corresponding assumption will be that the maximal synthesis rate is $b 1+b 2$, with gene 1 in charge of the rate $b 1$ and gene 2 in charge of the rate $b 2$. If both gene 1 and gene 2 are activated, the target gene will be inhibited.

To our best knowledge, there is no general conclusion for whether multiplicative or additive model is more accurate. This may depend on specific biological systems. Indeed both additive and multiplicative forms of Hill functions have been used in describing gene regulations [16,18,28,34-37]. In fact, recent studies suggested that it is the network topology that determined the operation principle of the networks, and additive Hill functions and multiplicative Hill functions may give similar conclusions [34,38]. In this work, we used additive Hill functions to construct our model. To simplify the modeling procedure, the parameter $a_{j i}, b_{j i}$ and $k_{i}$ were all fixed for different gene variables or regulations (written as $a, b$ and $k$ in the following text), individually. The degradation rate is set as $k=1$. To describe the nonlinear interactions among genes of the system, the Hill coefficient $n$ is usually set larger than 2, e.g., from 2 to $6[28,33,39]$. Here we take $n=5$ as an example [33] (details for the choice of Hill coefficient are discussed in the Appendix). Therefore, once the parameter $a, b$, and $s_{j i}$ are set, we can calculate the steady states of the dynamical system given the initial condition of the system, by solving the ODEs numerically.

After constructing the dynamical model, one issue remains, which is how to determine the parameter values. Here, the parameters that need to be determined include $a$ (activation constant, same for all activation regulations), $b$ (repression constant, same for all inhibition regulations), and $s_{j i}$ (for the interaction from $j$ to $i$ ), so the total number of parameters is $2+71=73$. The parameters of the model should be determined so that the mathematical model can generate the simulation data as close as to the experimental data. That is to say, the computational model should be able to generate three stable states that are consistent with experimental cell states (MP, B-cell and Mac), and the expression level of each gene at corresponding stable state should be approximately consistent with the gene expression value from experimental data.

\section{B. Parameter inference based on steady-state expression data}

To infer the model parameters, we developed a gradient descent algorithm to optimize the parameters in our computational model. According to the Euler method, for a $N$-dimensional system (e.g., including $N$ gene variables, here $N$ is 23$)$ with an initial condition $\left[x_{1}(0), x_{2}(0), \ldots, x_{N}(0)\right]$, we can calculate the state at time $t_{k+1}$ as follows:

$$
\begin{aligned}
x_{i}\left(t_{k+1}\right) & =x_{i}\left(t_{k}\right)+h f_{i}\left[\boldsymbol{x}\left(t_{k}\right)\right] \\
& =x_{i}\left(t_{k}\right)+h\left[\sum_{j \in M_{i}} \frac{a_{j i} x_{j}^{n}}{s_{j i}^{n}+x_{j}^{n}}+\sum_{j \in M_{i}^{\prime}} \frac{b_{j i} s_{j i}^{n}}{s_{j i}^{n}+x_{j}^{n}}-k_{i} x_{i}\right] .
\end{aligned}
$$

Here $h$ represents the time step in the Euler method, $t_{k}=k h$ represents the discretized time, and $f_{i}$ describes the regulation force on the $i$ th gene. Our aim is to estimate parameters that can generate simulation results consistent with experimental data. Such aim includes two aspects. First, the number of the stable states in our model should be as least three (corresponding to three cell states observed in experiments in HSC system, including MP, B-cell, and Mac). Second, the 
TABLE I. Pseudocode of the parameter inference algorithm

\section{Algorithm: Parameter inference}

Initializing: time interval $h$, learning rate $l$, maximum optimization steps $k_{\max }$, initial model parameters $a, b, s_{i j}$, initial cell state $\boldsymbol{x}^{\alpha}(0)$ (here we chose experiment data $\boldsymbol{y}^{\alpha}$ as initial cell state)

For $k=1,2, \ldots, k_{\max }$, do:

1: Calculate steady-state expression vector $\boldsymbol{x}^{\alpha}$, and the gradient $\frac{\partial \boldsymbol{x}^{\alpha}}{\partial \lambda}$

2: Calculate the gradient of loss function $\frac{\partial L}{\partial \lambda}$

3: Update parameters according to $\lambda=\lambda-l \cdot \frac{\partial L}{\partial \lambda}$

End for

Return $\lambda$

expression level of each gene in our model should be approximately consistent with the experimental data. Thus, the loss function $L$ for the optimization is defined as follows:

$$
L(\boldsymbol{\lambda})=\sum_{\alpha} \sum_{i}\left(x_{i}^{\alpha}-y_{i}^{\alpha}\right)^{2},
$$

where $\lambda$ represents the parameters of the model $\left(a, b\right.$, and $\left.s_{i j}\right)$, and $\alpha$ represents cell state (MP, Mac, B-cell). $x_{i}^{\alpha}$ represents the gene expression value of the $i$ th gene for the cell state $\alpha$ in the model, while $y_{i}^{\alpha}$ represents the gene expression data of the $i$ th gene for the cell state $\alpha$ from experiments. The loss function describes the difference of each gene between the simulation and the experimental data. By differentiating both sides of the Eq. (2) with respect to parameter $\lambda$, we can obtain

$$
\begin{aligned}
\frac{\partial x_{i}\left(t_{k+1}\right)}{\partial \lambda} & =\frac{\partial x_{i}\left(t_{k}\right)}{\partial \lambda}+h \frac{\partial f_{i}\left(\boldsymbol{x}\left(t_{k}\right), \lambda\right)}{\partial \lambda} \\
& =\frac{\partial x_{i}\left(t_{k}\right)}{\partial \lambda}+h\left[\sum_{j} \frac{\partial f_{i}}{\partial x_{j}} \cdot \frac{\partial x_{j}\left(t_{k}\right)}{\partial \lambda}+\frac{\partial f_{i}}{\partial \lambda}\right] .
\end{aligned}
$$

In this way, the gradient $\frac{\partial L}{\partial \lambda}$ can be calculated, and the parameters can be updated at a rate of $l$ as follows:

$$
\begin{aligned}
\lambda_{\text {new }} & =\lambda-l \frac{\partial L}{\partial \lambda} \\
& =\lambda-l 2\left[\sum_{\alpha} \sum_{i}\left(x_{i}^{\alpha}-y_{i}^{\alpha}\right) \frac{\partial x_{i}^{\alpha}}{\partial \lambda}\right] .
\end{aligned}
$$

Following Eq. (4), we can calculate $\frac{\partial x_{i}^{\alpha}}{\partial \lambda}$, update the parameters according to Eq. (5), and obtain the optimized parameters. The pseudocode of the algorithm is summarized in Table I (see the Appendix and Figs. 7 and 8 for an example of parameter inference for a two-variable gene network model and more details).

We applied our optimization algorithm to the HSC modeling (Please see the Appendix and Fig. 9 for details). The modeling results from parameter optimization are shown in Fig. 2 (right, models 1 and 2), as compared with experimental data (Fig. 2, left). All the expression values were normalized to the range of 0 to 1 . Here, models 1 and 2 represent the results of optimization for parameter inference, starting from the best initial parameter values and the second best initial parameter values (from random parameter search), respectively. With the optimized model parameters, the model can generate three steady states corresponding to the three cell states from experiments (for both model 1 and model 2). The comparison for the gene expression value between the model 1 and model 2 as well as experimental data showed that the result from the second best initial parameter set (model 2) gives a similar result as the one from the best initial parameter set (model 1) in terms of fitting experimental data, which supports the validity and robustness of our optimization algorithm. Interestingly, the model with the optimized parameters also generate a fourth state (marked as IM), with high expression level for both genes Cd19 and Mac1, which is not similar to any of three cell states from experimental data. We believe that it may play critical roles in HSC differentiation. We will make further discussions on this intermediate state in following analysis. Since the models 1 and 2 give similar results, we will use the results of model 1 to do the following analysis.

\section{Potential landscape and the minimum action path of the HSC differentiation and transdifferentiation}

To study the global stability and stochastic dynamical properties of HSC differentiation system, we aim to map out its potential landscape. Based on the gene regulatory network of HSC differentiation, we were able to write down the ODEs. The steady-state probability distribution of the network can be calculated by the self-consistent approximation approach $[18,28,29,40]$, and then the potential landscape can be constructed by $U=\ln \left(P_{s S}\right)[13,28]$ (see Methods for the details on how to calculate the landscape). Here $U$ represents the dimensionless potential, while $P_{s s}$ represents the steady-state probability distribution of the system. Since the steady-state probability distribution we obtained here has high dimensions, which is difficult to visualize, we selected Mac1 and $\mathrm{Cd} 19$, the marker genes of Mac cells and B-cells, as the coordinates for projection by integrating all the other variables. This makes it possible to visualize the landscape in a three-dimensional picture [Fig. 3(a)]. The blue region represents lower potential or higher probability, while the yellow region represents higher potential or lower probability. Four basins emerged on the potential landscape, characterizing four stable cell states including MP, Mac, B-cell, and an intermediate state IM. For the IM state, both $\mathrm{Cd} 19$ and Mac1 are highly expressed. As we will discuss in the following analysis, this intermediate state plays a critical role in the transdifferentiation.

To fully understand the process of differentiation and transdifferentiation and discover the role of the intermediate state, we studied the transition paths between different stable states or attractors. Here we computed the kinetic transition paths between different attractors by minimizing the transition actions through Freidlin-Wentzell theorem (see methods for how to quantify the transition paths) [41-43]. The MAPs for different transitions are shown in Fig. 3 on the potential landscape. White lines denote the transitions for differentiation (including the MAPs from MP to B-cell and Mac), purple lines denote transitions for reprogramming (including MAPs from B-cell and Mac to MP), and green lines denote transitions for transdifferentiation (including MAPs from B-cell to Mac and from Mac to B-cell). We can see the transdifferentiation between Mac states and B-cell state (green lines) both go through the intermediate state IM instead of through MP state or making direct transitions. This indicates that the IM state we identified here is an intermediate state in the 
(a)

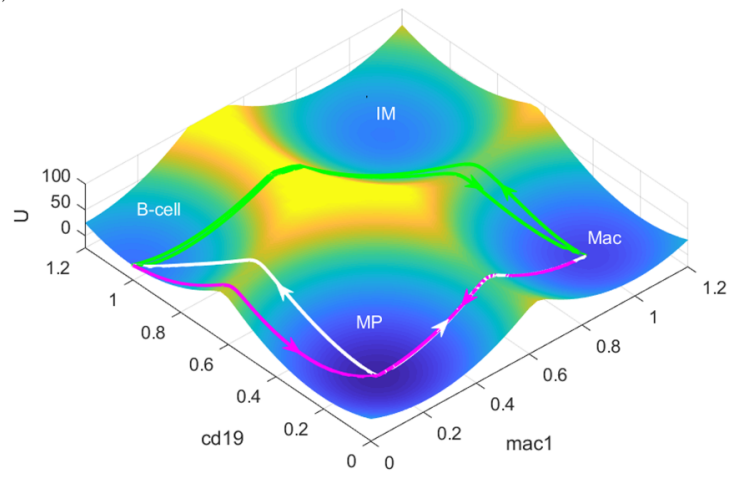

(b)

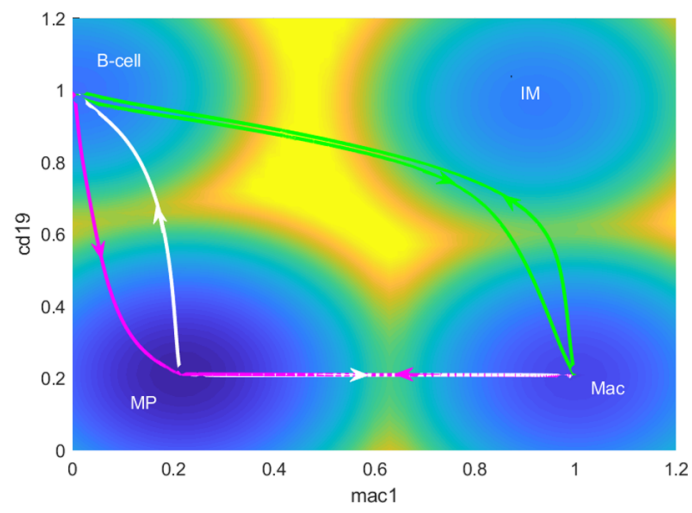

FIG. 3. The landscape and corresponding MAPs for the HSC network shown in three-dimensional (a) and two-dimensional (b) figures. White lines represent the MAPs for differentiation (from MP state to B-cell state and Mac state). Purple lines represent the MAPs for reprogramming (from B-cell state and Mac state to MP state). Green lines represent the MAPs for transdifferentiation (from B-cell state to Mac state and from Mac state to B-cell state). The blue area around the steady states represents high probability or low potential, and the yellow area represents low probability or high potential. Here MP represents multipotent progenitors, Mac represents macrophages, and IM represents intermediate state.

transdifferentiation process between Mac state and B-cell state. It also suggests that the intermediate state IM may play critical roles in transdifferentiation processes, which is consistent with previous work on the roles of intermediate states for embryonic stem cell differentiations [44].

To see the transition path in high-dimensional space with multiple genes, we quantified the transition paths for the 23-dimensional system. Here we considered the transdifferentiation process between B-cell state and Mac state. We displayed a continuous representation of transition paths by normalizing the gene expression [Figs. 4(a) and 4(b)]. However, it would be difficult to define when genes switch on or off in continuous representation. Therefore, we also presented a binary representation by discretizing the gene expression value to 1 (on state) or 0 (off state). Since the gene expression values were normalized to the range from 0 to 1 , here we chose 0.5 as the threshold value to discretized the gene expression value. The binary paths are shown in Figs. 4(c) and $4(\mathrm{~d})$. We take the transdifferentiation path from B-cell state to Mac state as an example to discuss gene switching mode in this stage. The transdifferentiation process starts with the switch-off of Gfil and switch-on of Cebpa, followed by the switch-on of Cebpb, Spi1, and Mac1. Then Egr1 switched on, Foxo1 switched off, and finally Cd19 and Mef2c switched off, which marks the end of the transition.

These results are consistent with several existing experimental observations. For example, Cebpa and Cebpb were confirmed to induce the transdifferentiation from B cells to Mac cells [1,4]. Egr1 and Gfi1 have opposite effects in inducing the differentiation of hematopoietic stem cells. The former will drive the differentiation toward Mac cells while the latter will drive the differentiation of B-cells [45]. Foxo1 is important in B-cell branching, which can inhibit Cebpa itself [8]. Mef2c plays a major role in the differentiation of HSCs [46].

\section{The discretized landscape from stochastic simulations shows consistent results with continuous landscape}

Because of the existence of intrinsic noises from limited number of molecules and external fluctuations from the environment [47-49], the dynamic changes of these cells are often complicated. Except the differentiation process, such as from stem cells to MP and from MP to B-cells or Mac cells, some other transition processes may also happen, including the reprogramming (a differentiated cell is converted to an undifferentiated stem cell), or transdifferentiation (e.g., the transition between B-cells and Mac cells). In above section, we constructed the landscape by projecting multidimensional results to certain low dimensions. To display the cell states and the transitions between different cell types in the complete state space, we discretized the expression level of the 23 marker genes to binary states (total $2^{23}$ cell states) [28]. To achieve this, we applied the Langevin simulations to obtain a discretized landscape by discretizing the expression level of each gene to 1 (on state) or 0 (off state). The Langevin equations describing the stochastic dynamics of the system take the following form:

$$
\frac{d x}{d t}=f[x(t)]+\zeta
$$

Here $\zeta$ is a Gaussian white noise term satisfying $\left\langle\zeta_{i}(x, t) \zeta_{j}(x, 0)\right\rangle=2 D \delta(t), D$ is the diffusion coefficient, and $\boldsymbol{f}$ is the driving force specified in Eq. (2).

Therefore, by running stochastic simulations from Eq. (6), we can obtain time series of the gene expression values of the system and transform them to binary form by setting cutoffs. Due to the inclusion of a Gaussian noise, the expression level of certain gene can be exceptionally large at one time point, which may lead to inaccurate results after normalization. So we did not do the normalization for the stochastic models and used the half of the theoretical largest expression value (from the model equations) as the cutoff for each gene. After binarization, we used marker genes to identify corresponding cell states. Specifically, if a state has Mac1 off and Cd19 off, it is identified as MP state. A state with Mac1 on and Cd19 off is identified as Mac state. A state with Cd19 on and Mac1 off is identified as B-cell state. Finally, a state with Mac1 on and $\mathrm{Cd} 19$ on is identified as the intermediate state IM. 
(a)

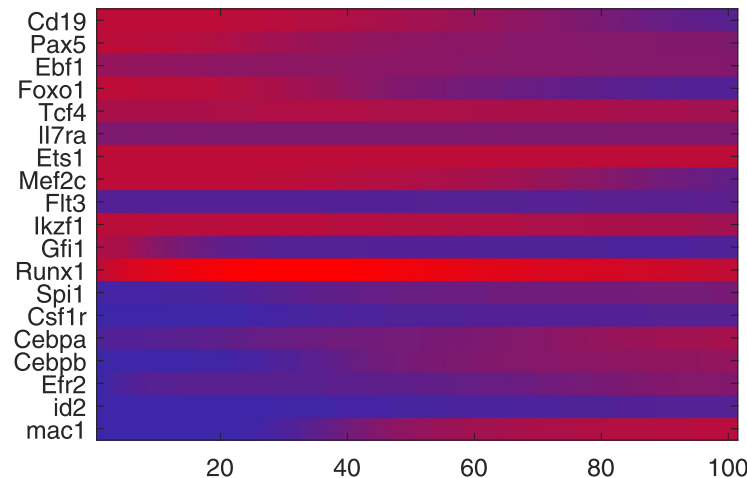

(c)

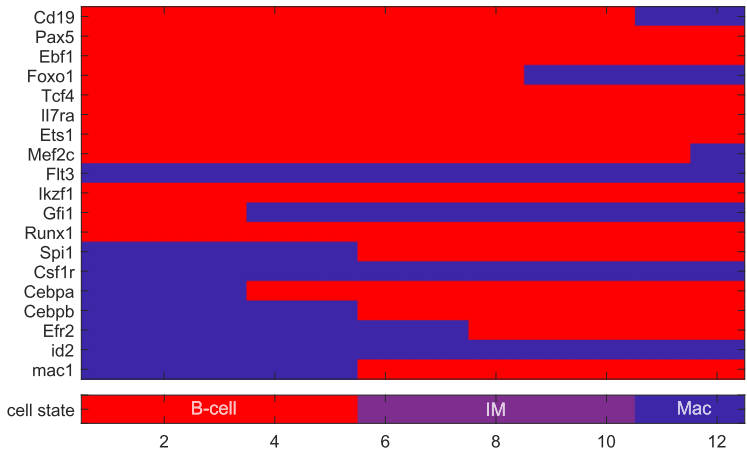

(b)

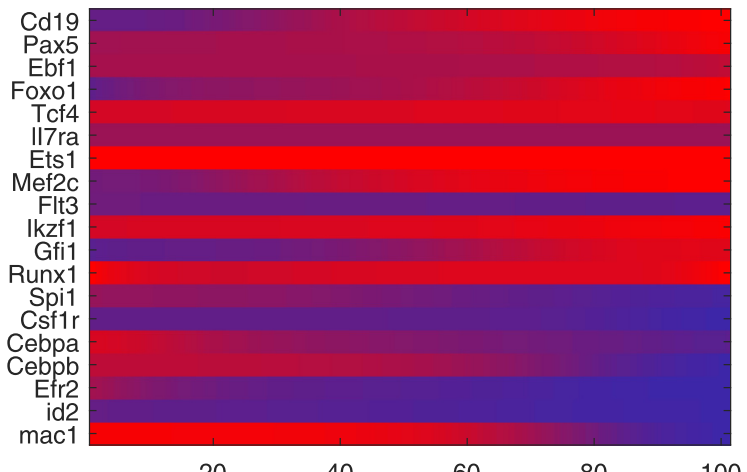

(d)

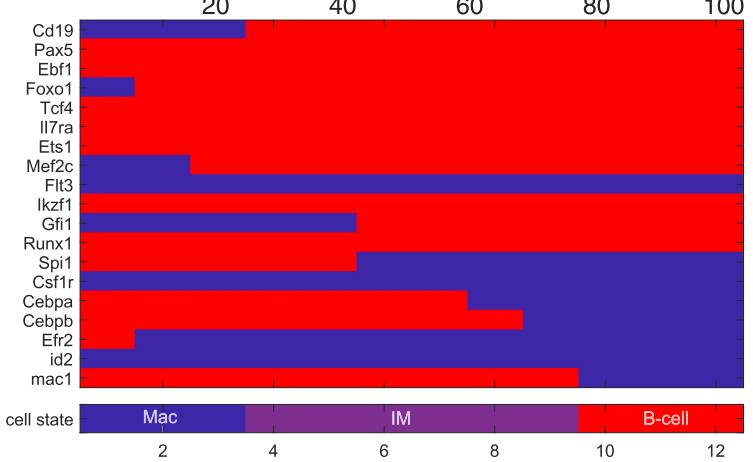

FIG. 4. The transition path from B-cell state to Mac state [(a) for continuous representation and (c) for discrete representation] and from Mac state to B-cell state [(b) for continuous representation and (d) for discrete representation] in terms of the activity of genes in HSC regulatory network. In continuous representation, the expression values of genes are normalized to the range from 0 to 1 , where red denotes 1 and blue denotes 0 . In discrete representation, the expression levels of genes are discretized into binary mode, where red denotes the gene is in on-state and blue denotes the gene is in off-state. The $x$ axis represents the time point along the transition path, including 12 time points. The last line of blocks indicates the cell state at corresponding time point. Red block denotes B-cell state, purple block denotes the intermediate state, and blue block denotes Mac state.

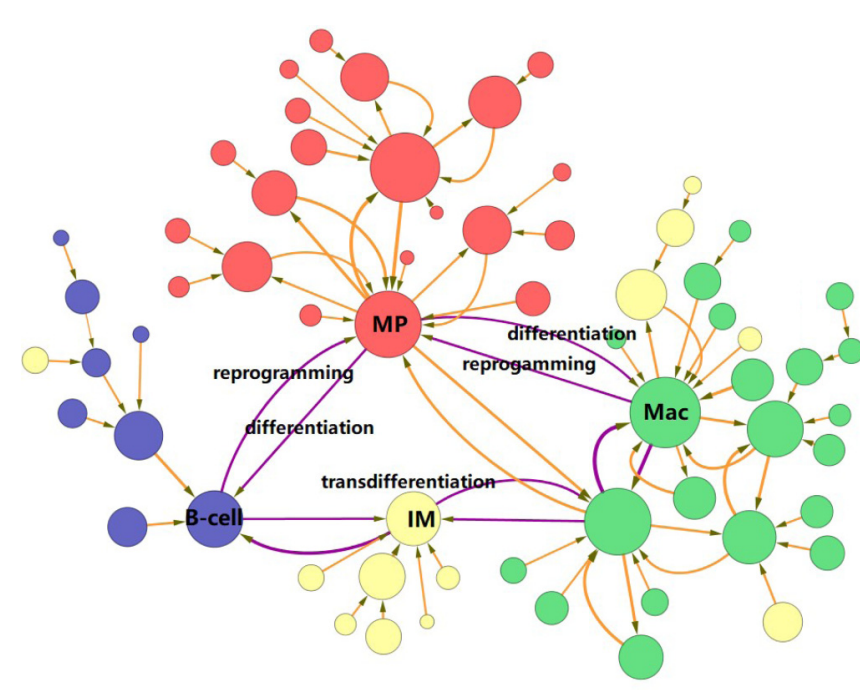

FIG. 5. Differentiation and transdifferentiation represented by 72 nodes (denoting cell states, characterized by expression levels of marker genes) and 84 edges (denoting transition paths). Red nodes represent the MP state, blue nodes represent the B-cell state, green nodes represent the Mac state, and yellow nodes represent the IM state. The size of the nodes and the width of the edges is proportion to the occurrence probability of the state and transition. Only nodes and edges with an occurrence over a threshold were shown on the figure for clearer depiction. MP represents multipotent progenitor, Mac represents macrophage, and IM represents intermediate state.
The result was depicted as a transition graph in Fig. 5. The different nodes represent different state and different links represent possible transitions. Only nodes and edges with an occurrence probability over a threshold were shown for clearer depiction. The sizes of the nodes and edges are proportional to the frequencies of the states and transitions in our simulations. It shows how the MP state differentiates into both B-cell and Mac, and more importantly, how Mac and B-cell transdifferentiate into each other through the intermediate state IM, which further demonstrates the importance of the intermediate state in HSC developmental process. A major conclusion of Fig. 5 is that in the discrete case with all gene considered, the models give the similar conclusion as the continuous case (Fig. 3).

\section{E. Discovery of key interaction links from sensitivity analysis on model parameters}

To investigate which regulations play critical roles in the cellular differentiation and transdifferentiation process of HSCs, we performed a global sensitivity analysis on the model parameters. As an example, here we focus on the transdifferentiation between B-cell and Mac. We changed the parameter of each regulation (including regulation strength $a, b$ or Hill function threshold $s_{j i}$ ), and calculated the relative variation of the transition action between the B-cell state and Mac state after parameter changes (corresponding to the barrier height between basins). A larger variation of the 
(a)

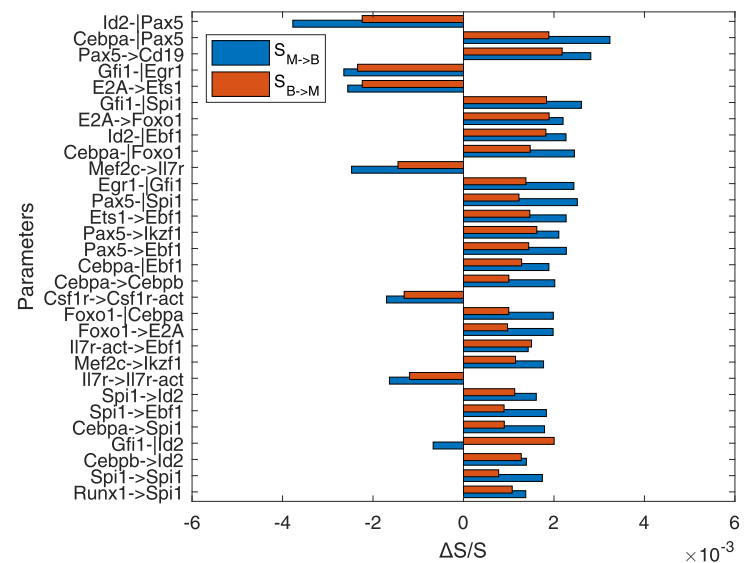

(c)

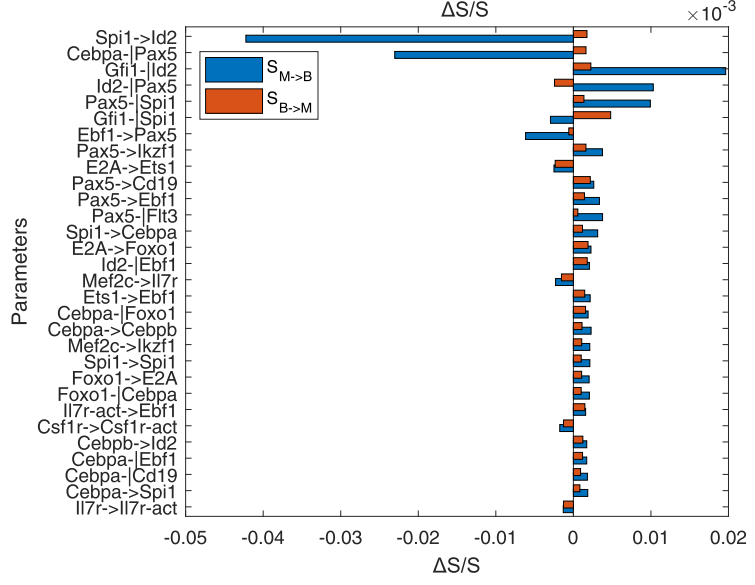

(b)

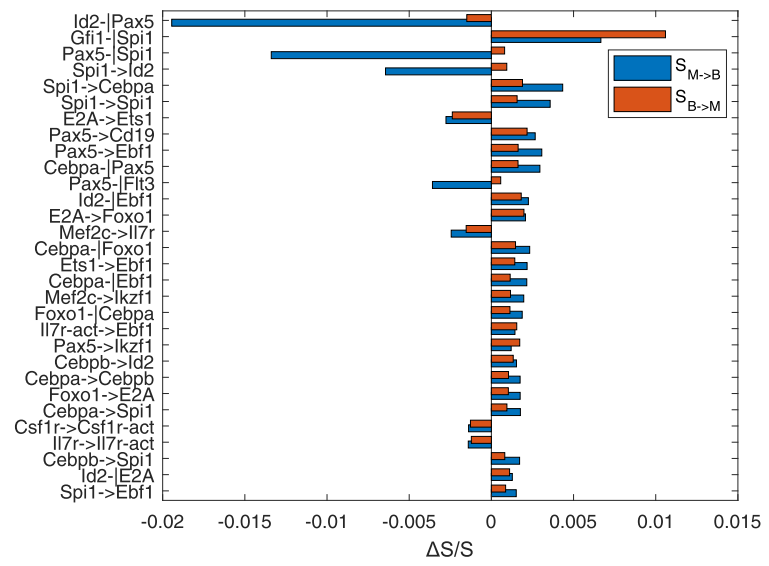

(d)

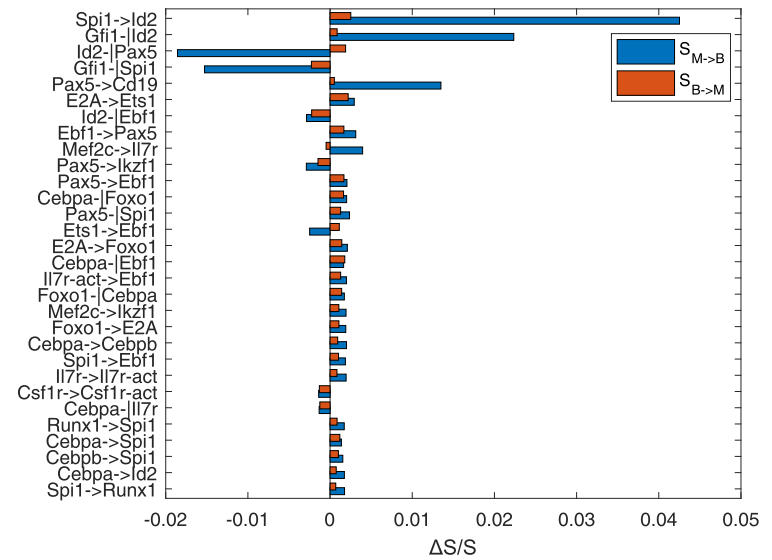

FIG. 6. Sensitivity analysis for the 71 key parameters on the transition action $\left(S_{M \rightarrow B}\right.$ and $\left.S_{B \rightarrow M}\right)$. The Y-axis represents the 71 parameters (only top 30 most influential regulations are shown, based on corresponding change of transition actions). The $x$ axis represents the relative variation of transition action after changing parameters $(\Delta S / S)$. Here, $S_{M \rightarrow B}$ represents the transition action from Mac state to B-cell state (blue bars), and $S_{B \rightarrow M}$ represents the transition action from B-cell state to Mac state (red bars). (a) Each parameter $s_{i j}$ is increased by $15 \%$ individually. (b) Each parameter $s_{i j}$ is decreased by $15 \%$ individually. (c) Each parameter $a / b$ is increased by $15 \%$ individually. (d) Each parameter $a / b$ is decreased by $15 \%$ individually.

transition action indicates a major impact of the regulation on transdifferentiation.

The result of the sensitivity analysis is shown in Fig. 6. Only regulations with top 30 largest variation of transition action are shown for clarity. The regulations of Id $-\dashv \operatorname{Pax} 5$, Cebpa $\dashv$ Pax 5 and Pax $5 \rightarrow \mathrm{Cd} 19$ have the highest relative variation of transition action among all the regulations when we increase threshold $s$ by $15 \%$, indicating these three regulations are most critical to the transdifferentiation process. Specifically, when the threshold $s$ of the regulation Id $2 \dashv \operatorname{Pax} 5$ is increased, the transition action from the Mac state to the B-cell state decreases relatively, which means that the attenuation of this regulation will promote the transition from Mac state to B-cell state. Similarly, the attenuation of the regulation Cebpa $-\mathrm{Pax} 5$ and $\mathrm{Pax} 5 \rightarrow \mathrm{Cd} 19$ may inhibit the transition from the Mac state to the B-cell state. The regulations Id2 $\dashv$ Pax5, Gfi1 $\dashv$ Spi1 and Pax5 $\dashv$ Spi1 have the largest variation of transition action when the threshold $s$ is decreased by $15 \%$. Though different from the most influential regulations when increasing parameter $s$ by $15 \%$, these three regulations are among the top 10 influential regulations. The sensitivity analysis that focuses on the parameter of activation and inhibition strengths showed similar results (Figs. 6(c) and 6(d)). For example, the regulations Spi1 $\rightarrow$ Id2, Gfi1 $\dashv$ Id2, and Id $2-$ Pax 5 are also among the top 30 largest relative variation of transition action when increasing and decreasing regulation strength $a / b$, and we see that for the most influential 20 regulations by increasing or decreasing regulation strength $a / b$, there is an overlap of 15 out of 20 regulations, which confirms that these regulations are critical to cellular transdifferentiation.

These results consistently indicate that some factors including Pax5, Cebpa, Spi1, and Id2 play key roles in transdifferentiation process, which is highly consistent with previous studies. Cebpa is a key gene that induces B cells to transdifferentiate into Mac cells, and the process of induced reprogramming includes significant inhibition of Pax5 [4]. Pax 5 plays a key role in the commitment of B-cell lineages. The knock-out or mutation of Pax 5 can cause cell cycle arrest [50]. Id2, as a transcription factor of inhibitor of differentiation, may inhibit the differentiation of B-cells based on its antagonism to Pax5 and E2A [51]. The Spil gene plays an important role in the development of B-cells and Mac cells [52]. Ikzf1 controls the differentiation of cells into B-cell lines, and its inhibition on Spi1 may deviate the development of cells into B-cells [53]. Though the regulation of Ikzf1 -1 Spi1 was not identified as critical regulation in our analysis, but Pax5, which binds to Ikzf1 at the same position, has a 
larger relative variation of transition action, which indicates that Pax $5 \dashv$ Spi1 is also a key regulation of the differentiation process [4].

\section{DISCUSSION}

In recent years, gene regulatory network models have been widely exploited to study cell fate decisions [16,28,37,39,54]. To study the cell fate decision mechanism of HSC differentiation, we constructed an ODE model describing the HSC differentiation process, based on the underlying gene regulatory network [8]. A critical challenge in gene network modeling is how to determine the model parameters, especially when the valid dynamical data is not available. In this work, we developed a parameter optimization method by combining gradient descent algorithm and random parameter search, based on static experimental data (steady-state data). This approach proved more effective and provided better result than random parameter search alone and has great potential in terms of parameter inference in gene network modeling. By using a two-dimensional gene network model as an example, we validated the efficacy of this approach for parameter inference.

We further applied our parameter inference approach to the HSC model. We found that the models after parameter optimization can fit well three stable cell states observed in experiments (MP, B-cell, and Mac). Interestingly, our model from parameter optimization also suggests a fourth stable state, i.e., a new cell state not yet observed in experiments. This new cell state has both high Cd19 and Mac1 expression, indicating a possibility of being an intermediate state. By further analysis based on transition path theory, we found that this new identified state corresponds to an intermediate state, which plays critical roles in transdifferentiation between B-cell and Mac. Many results have illustrated the importance of intermediate states in previous studies related to stem cell differentiation, epithelial-mesenchymal transition (EMT) and cancer-immune system [44,54-57].

The potential landscape we quantified from gene network models offers us a global view of cell fate decision process in HSC differentiation. MP, B-cell, and Mac, along with another intermediate state are depicted as basins on the landscape surface. By calculating the transition path between different attractor states, we showed that for the transdifferentiation process cells mostly go through the new intermediate state that we identified (Fig. 3). This emphasizes the importance of the intermediate state in cell fate determinations. These results are further confirmed by a discretized landscape based on stochastic simulations. From the potential landscape and transition path, we see different differentiation states of HSCs in a more intuitive and physical way and identify the sequence of gene switch in the process of differentiation and transdifferentiation.

To identify the critical factors in differentiation and transdifferentiation process, we performed a global sensitivity analysis on model parameters. Our analysis revealed key regulators in transdifferentiation process. For example, we revealed the key role of Spi1 $\dashv$ Cebpa in the process of transdifferentiation, which is consistent with previous study [8]. We

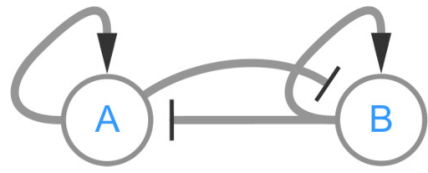

FIG. 7. A two-gene network with self-activation and mutual inhibition. A and B represent two genes. $\rightarrow$ represents activation, and $\dashv$ represents inhibition.

also found specific key regulations involved in the process of transdifferentiation and differentiation (such as Cebpa $-\mathrm{Pax} 5$, Foxo1 $\dashv$ Ebf1 and $\mathrm{Cebpa} \rightarrow \mathrm{Cebpb})$.

In this work we used additive Hill functions to construct the gene regulatory model, which may facilitate the computation of the gradient descent algorithms. How to implement the optimization method of gradient descent to models with multiplicative Hill functions requires further explorations. In our model, for simplicity the coefficient $a$ and $b$ are fixed for all regulations (each $a / b$ corresponds a regulation). A common way of parameter inference in this type of model is random parameter search, which may be difficult to carry out if there are too many parameters in the model. Therefore, we used this assumption to reduce the number of parameters and simplify the models, since we will use the result of a random parameter search as our initial set of parameters for gradient descent algorithm. The feasibility of such method in a more complicated model (involving much more parameters) warrants further explorations.

To test the robustness of our approach, we performed the parameter optimization starting from different initial parameter values. For a simplified two-dimensional system, our approach displayed good reproducibility for the parameter optimization from different initial parameter values (Fig. 8). This might be because for a simplified low-dimensional system the global minimum is easy to find. However, for the HSC system different initial parameter values lead to different results for parameter inference. Considering the high dimensionality of the complex HSC system, it would be understandable that the global minimum may be difficult to find from a gradient descent algorithm. The optimized parameter sets starting from different initial parameter values might correspond to different local minimum although they can all fit experimental data well. Therefore, for a high-dimensional complex system, it may be hard to find the unique solution (global minimum) for the parameter inference using our approach. But since our purpose is to identify the parameters which fit experimental data well, the local minimum provides a possible solution already, which is what our approach is trying to address.

Of note, recently Weinreb et al. concluded that the assumption of a potential landscape imposes symmetry on the gene regulatory network [58]. This conclusion was based on an equilibrium landscape theory, i.e., the deterministic driving force is assumed to be a gradient field. The HSC gene regulatory network studied in our work is not a symmetrical network. In fact, a landscape and flux theory for the nonequilibrium system has been developed and applied to many biological networks including both symmetrical and 
(a)

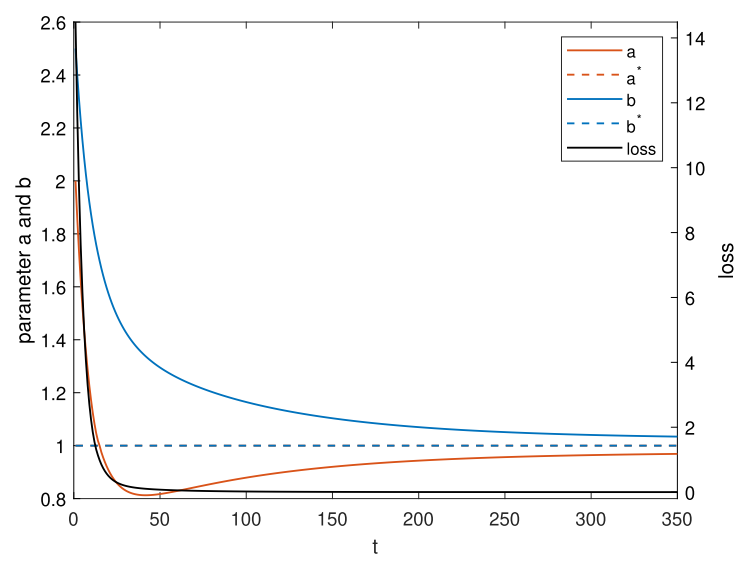

(b)

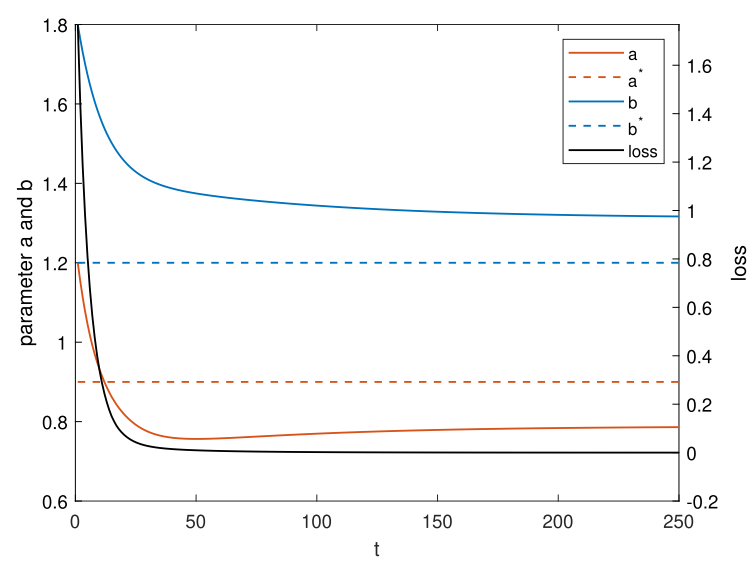

(c)

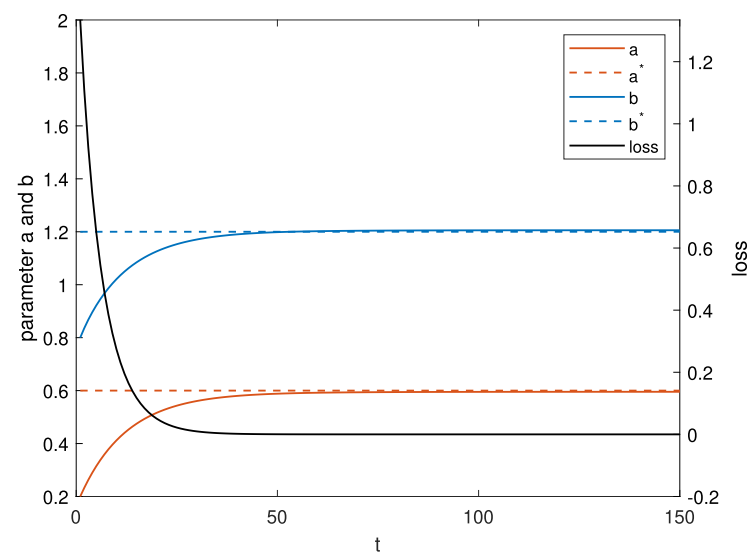

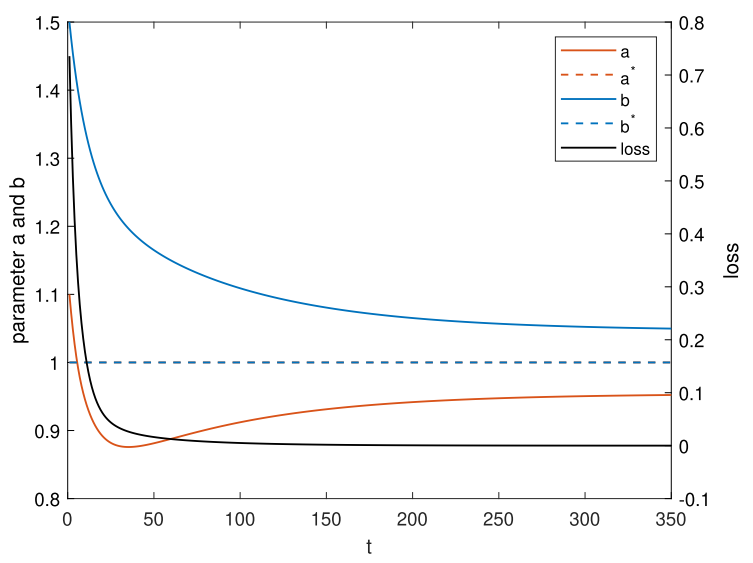
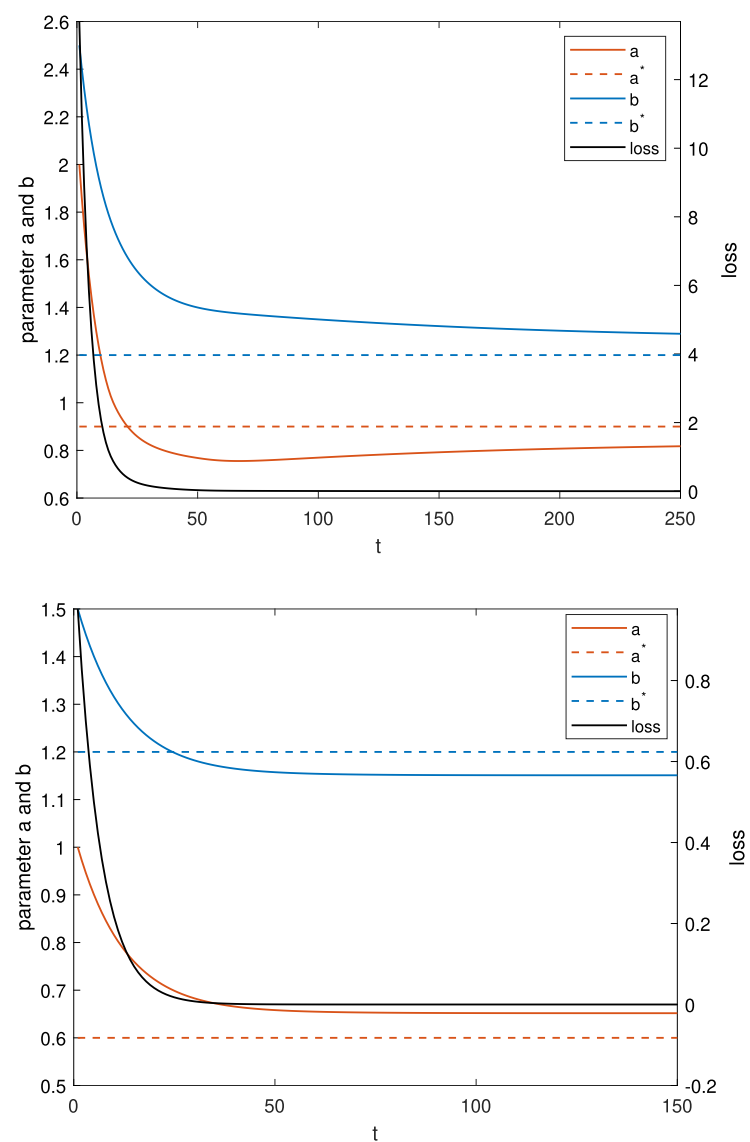

FIG. 8. The change of parameter $a, b$ and the loss during the process of optimization under three sets of parameters (a) tristable system: $a=1, b=1, s_{i j}=0.5$, (b) tristable system: $a=0.9, b=1.2, s_{11}=0.5, s_{12}=0.6, s_{21}=0.3, s_{22}=0.4$, and (c) bistable system: $a=0.6$, $\left.b=1.2, s_{11}=0.5, s_{12}=0.6, s_{21}=0.3, s_{22}=0.4\right)$. In each case two different tests (left and right) were done with different initial parameter values. Red (blue) solid lines represent the parameter $a(b)$ during optimization, and red (blue) dashed lines represent the actual parameter value of the original model $a^{*}\left(b^{*}\right)$. The black solid lines represent the loss. In (a), the red and blue dashed lines overlap since the original parameter $a^{*}$ and $b^{*}$ are the same in this model.

asymmetrical gene networks [16,18,28,59]. An interesting question arises as to what is the relationship between the potential landscape concept and the symmetrical characteristics of the network. For example, previous work showed that even in a simple symmetrical gene network model, the driving force does not correspond to a pure gradient term, and the rotational flux term still plays a critical role [16].
In summary, our work provides a general approach for parameter inference of gene network models based on static (steady-state) expression data. The results of potential landscape and transition paths of the HSC network facilitate our mechanistic understanding of the HSC differentiation and transdifferentiation, which also reveal the importance of intermediate states in HSC development. 


\section{METHODS}

\section{A. Parameter inference method}

We aim to infer the model parameters that can replicate the experimental data in HSC differentiation. A random parameter search was done at first to determine the initial parameters for optimization. Specifically, an approximate range of each parameter based on previous work on gene network modeling (from 0 to 2 for $a, b, s_{i j}$ ) [28,33] was determined at first. Further, by discretizing each parameter into 20 groups (a total of $20 * 20 * 20$ sets of parameters) and making simulations with each set of parameters, we can choose a parameter range that allows the system to generate multiple steady states ( $a$ from 1.2 to $1.6, b$ from 0 to 0.5 , and $s$ from 0.5 to 1.5 ) and begin the random parameter search. The best result from 100000 tests are chosen as the initial parameters for optimization. The gradient descent optimization was then conducted to infer the parameters that can generate simulations consistent with the experimental data. An example of the optimization procedure for a two dimensional gene network case can be found in the Appendix.

\section{B. Self-consistent mean-field approximation}

The time evolution of a dynamical system is governed by the probabilistic diffusion equations. The system state can be represented by $P(X, t)$, where $\boldsymbol{X}=\left(X_{1}, X_{2}, X_{3}, \ldots, X_{N}\right)$ represents expression level of $N$ different genes in the system. We then have a system of $N$ coupled partial differential equations. However, the diffusion equation is difficult to solve when the dimension of the system is large. Following a selfconsistent approximation [18,28,29], by assuming that the different genes in the system are weakly correlated, we can split the probability distribution into the products of individual probabilities: $P(\boldsymbol{X}, t) \sim \prod_{i}^{N} P\left(X_{i}, t\right)$. In this way, we can reduce the dimension from $M^{N}$ to $M N$ ( $M$ is the possible state number for single gene (For instance, in a binary system $M$ $\in\{0,1\}$, and in our HSC network case, $M$ is chosen to be 1000). Next, we start from the moment equations and make an assumption of specific probability distribution based on the physical argument. In this work, we take the Gaussian distribution as an approximation, meaning that we need to calculate two moments, i.e., the mean and the variance. When the diffusion coefficient $D$ is small, the moment equations can be approximated to $[60,61]$

$$
\begin{aligned}
& \frac{d \bar{x}(t)}{d t}=f[\overline{\boldsymbol{x}}(t)], \\
& \frac{d \sigma(t)}{d t}=\sigma(t) \mathbf{A}^{\mathbf{T}}(t)+\mathbf{A}(t) \sigma(t)+2 D[\overline{\boldsymbol{x}}(t)] .
\end{aligned}
$$

Here, $\overline{\boldsymbol{x}}(t), \sigma(t)$ and $\mathbf{A}(t)$ are vectors and matrix. The elements of matrix A are specified as: $A_{i j}(t)=\frac{\partial f_{i}[x(t)]}{\partial x_{j}}$. The matrix $D[\overline{\boldsymbol{x}}(t)]$ is the diffusion matrix, specified as $D d[\overline{\boldsymbol{x}}(t)] d[\overline{\boldsymbol{x}}(t)]^{T}$, where $d[\overline{\boldsymbol{x}}(t)]$ is a $N * N$ matrix and $D$ the diffusion coefficient. Here, we focus on the external noise case, so $D$ is a constant, and $d[\overline{\boldsymbol{x}}(t)]$ is identity matrix. By solving the steady-state solution of these differential equations, we can obtain the mean and variance, $\overline{\boldsymbol{x}}(t)$ and $\sigma(t)$. Here, we only consider the diagonal elements of $\sigma(t)$ due to the mean-field approximation. Therefore, we can approximate the probability distribution for each gene variables from the Gaussian approximation:

$$
P(\boldsymbol{x}, t)=\frac{1}{(2 \pi)^{\frac{N}{2}}|\sigma(t)|^{\frac{1}{2}}} e^{\sum_{i=1}^{N}-\frac{\left[x_{i}(t)-\bar{x}_{i}(t)\right]^{2}}{2 \sigma_{i i}(t)}} .
$$

Here, $\overline{\boldsymbol{x}}(t)$ and $\sigma(t)$ are the solutions of Eq. (7). From the mean-field approximation, we can extend this formulation to the multidimensional case by assuming that the total probability is the product of each individual probability for each variable. The probability distribution obtained above corresponds to one stable state. If the system has multiple steady states, then there should be several probability distributions localized at each basin with different variances. Therefore, we can express the total probability as the weighted sum of all these probability distributions. For example, for a bistable system, the probability distribution takes the form: $P(\boldsymbol{x}, t)=w_{1} P_{1}(\boldsymbol{x}, t)+w_{2} P_{2}(\boldsymbol{x}, t)$. The weighting factors $\left(w_{1}, w_{2}\right)$ (satisfying $w_{1}+w_{2}=1$ ) characterize the relative sizes of different basin of attraction. We determine the weights $w_{i}$ by giving a large number of random initial values to the ODEs, and then collect the statistics from all these different solutions. Finally, we can construct the potential landscape by: $U(x)=-\ln P_{s s}(x)[18,28]$, with $P_{s s}$ representing the steady-state probability distribution.

\section{Minimum action paths}

In the cell, there exists intrinsic noise arising from statistical fluctuations of the finite number of molecules, and external noise originating from highly dynamical and inhomogeneous environments. The intrinsic and extrinsic noise can be critical to the dynamics of the system [47-49]. Here, for HSC differentiation system, we focus on the influence of external noise. A dynamical system in the fluctuating environments can be described by: $\dot{\boldsymbol{x}}=\boldsymbol{F}(\boldsymbol{x})+\zeta$, where $\boldsymbol{x}=$ $\left(x_{1}(t), x_{2}(t), \ldots, x_{N}(t)\right)$ represents the vector of the relative gene expressions, and $\boldsymbol{F}(\boldsymbol{x})$ is the vector for the driving force of chemical reaction. $\zeta$ is Gaussian noise term whose autocorrelation function is $\left\langle\zeta_{i}(\boldsymbol{x}, t) \zeta_{j}(\boldsymbol{x}, 0)\right\rangle=2 D \delta(t)$, and $D$ is diffusion coefficient matrix.

Following previous works based on the Wentzell-Freidlin theory $[41,42,54]$, the most probable transition path from attractor $i$ at time 0 to attractor $j$ at time $\mathrm{T}, \phi_{i j}^{*}(t), t \in[0, T]$, can be calculated by minimizing the action functional over all possible paths:

$$
S_{T}\left[\phi_{i j}\right]=\frac{1}{2} \int_{0}^{T}\left|\dot{\phi_{i j}}-\boldsymbol{F}\left(\phi_{i j}\right)\right|^{2} d t .
$$

This optimal path is called minimized action path (MAP). We calculated MAPs numerically by applying the minimum action methods used in Ref. [42].

\section{ACKNOWLEDGMENTS}

C.L. is supported by the National Key R\&D Program of China (Grant No. 2019YFA0709502) and the National Natural Science Foundation of China (Grant No. 11771098). The MATLAB implementation of HSC landscape and parameter inference approach is available at Ref. [62]. 


\section{APPENDIX}

\section{The selection of hill coefficient}

The Hill coefficient $n$ is usually set larger than 2 to describe the cooperativity for gene regulations $[28,33,39]$. We chose $n=5$ in our model as an example to describe the cooperativity for gene regulations and nonlinearity. To see a wide range of choice for Hill coefficient, we also made simulations with Hill coefficient $n$ ranging from 3 to 8 to compare the difference between the choice of Hill coefficient. With the parameter set obtained from our optimization approach, the system maintained to generate four steady states when $n=4-8$ (although there are differences in specific expression value), but failed to keep even bistable when $n=3$. It confirmed that Hill coefficient is crucial for producing multistability. Therefore, in this work the choice of Hill coefficient does not make a significant difference when $n=4-8$ in terms of multistability.

\section{Parameter inference for a simplified two-variable network}

To verify the effectiveness of the gradient descent method, we introduced a simplified two-dimensional gene network model, of which the regulatory network is shown in Fig. 7. We are able to write the ODEs that describe the system as follows:

$$
\begin{aligned}
& \frac{d A}{d t}=b \frac{s_{21}^{n}}{s_{21}^{n}+B^{n}}+a \frac{A^{n}}{s_{11}^{n}+A^{n}}-k A \\
& \frac{d B}{d t}=b \frac{s_{12}^{n}}{s_{12}^{n}+A^{n}}+a \frac{B^{n}}{s_{22}^{n}+B^{n}}-k B
\end{aligned}
$$

where the parameter setting is the same as in the main text. Hill coefficient $n$ and degradation rate $k$ were set to 5 and 1 , respectively, same as the parameter setting in the main text.

We first generate simulation data given parameter values $\left(a, b\right.$, and $\left.s_{i j}\right)$, and then we apply our parameter optimization approach to this model to infer the original parameters $a, b$, and $s_{i j}$. Without loss of generality, we tested it in three sets of parameters as shown in Fig. 8 (case (a) and (b) is for a tristable system, and case (c) is for a bistable system). The initial parameter value for the optimization should satisfy the constraint that the model can generate the same number of steady states as the original model. Two tests with different initial parameter values were performed to examine the stability of the optimization algorithm (shown in Fig. 8, left and right). The optimization results are shown in Fig. 8, and details of the result are shown in Table II.

In all tests the value of the loss decreased vastly (to a final loss less than $10^{-4}$ ) during the process of the optimization, which means the steady-state values from the model are very close to the actual expression value, indicating a success of the gradient descent method in parameter inference. We found that the loss value decreases to zero in all three models [Figs. 8(a), 8(b), and 8(c)], which indicates that the optimization method is robust in different models including tristable and bistable system. Our results also showed that the optimization results do not depend on the choice of initial values of parameters, as long as initial parameter sets can generate the same number of steady states as the original model (Fig. 8 left and right). This demonstrates the robustness
TABLE II. The relative deviation of regulation strength and the loss in all six tests corresponding to Fig. 8. Cases represent different sets of parameters (row in Fig. 8). The left and right tests in each case denote two initial parameters (column in Fig. 8).

\begin{tabular}{lcccc}
\hline \hline Cases & Tests & $\begin{array}{c}\text { Relative } \\
\text { deviation of } a\end{array}$ & $\begin{array}{c}\text { Relative } \\
\text { deviation of } b\end{array}$ & Loss \\
\hline (a) & Left & $-2.27 \%$ & $2.29 \%$ & $<1 \times 10^{-4}$ \\
& Right & $-4.60 \%$ & $4.63 \%$ & $<1 \times 10^{-5}$ \\
(b) & Left & $-12.44 \%$ & $9.32 \%$ & $<1 \times 10^{-5}$ \\
& Right & $-7.34 \%$ & $5.64 \%$ & $<1 \times 10^{-5}$ \\
(c) & Left & $8.60 \%$ & $-4.08 \%$ & $<1 \times 10^{-4}$ \\
& Right & $-0.75 \%$ & $0.48 \%$ & $<1 \times 10^{-4}$ \\
\hline \hline
\end{tabular}

of our optimization approach under different initial values of parameters.

Meanwhile, we see that the optimized parameter $a$ and $b$ both approach the real value in all tests with a small difference (Table II). This demonstrates that our parameter inference approach can recover the real value of key parameters $(a$

(a)

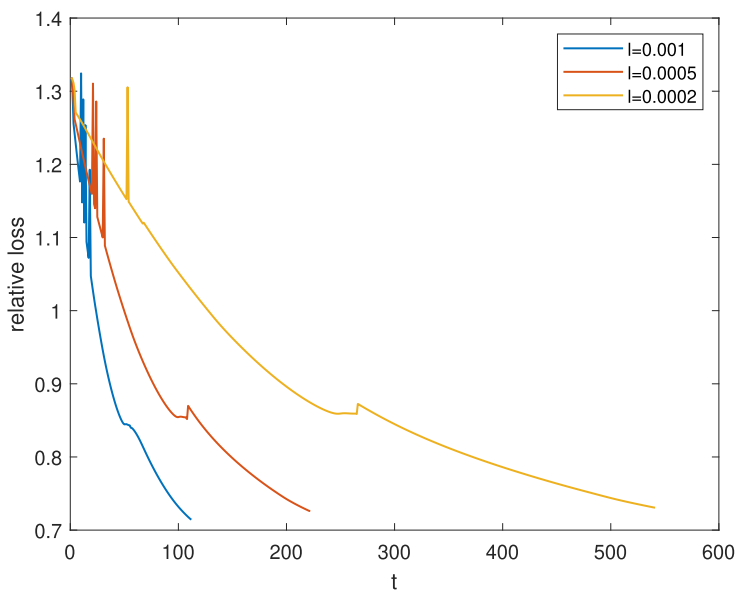

(b)

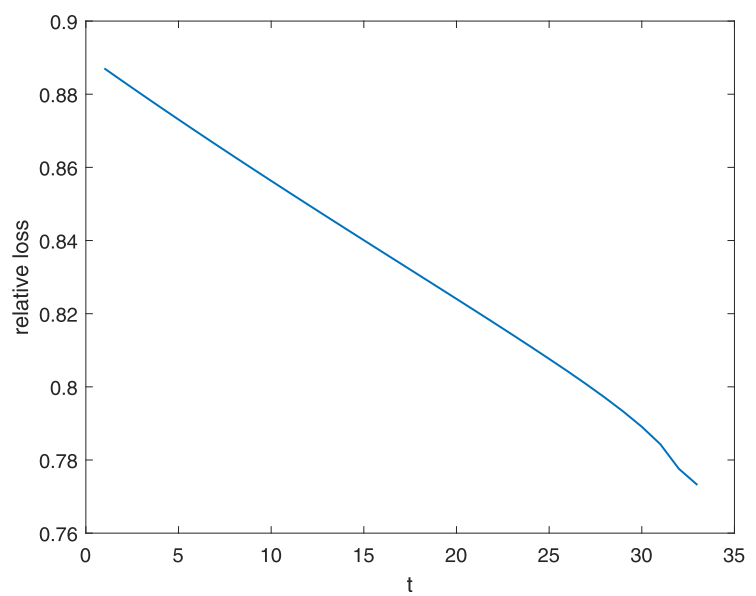

FIG. 9. The change of loss during optimization with different learning rate $l$, starting from (a) the best initial parameter values (from random parameter search), and (b) the second best initial parameter values. The $x$ axis represents the steps of optimization. The $y$ axis represents the relative loss. Lines of different color denote tests with different choice of learning rate $l$. 
and $b$ ) in current two-gene model, which provides further support to the reliability of our method. We also notice that the optimized parameter $s$ is not close to its real value (highly related to the choice of initial values). This might be due to its less crucial influence on the expression value of steady state, which warrants further explorations in future work.

\section{Estimating parameters in the hsc differentiation and transdifferentiation model}

Before we apply the gradient descent algorithm to the high dimensional HSC system, there are several problems remaining to be solved. First is the choice of initial values of parameters in the system. A set of parameters that can obtain at least three steady states is necessary in the optimization method. Otherwise there is a chance that one steady state is considered as two different cell states, which may lead to a failure in parameters optimization.

Another problem is the choice of the initial state of the system. Here, for simplicity, we chose the gene expression value from experimental data as the initial state of the system for parameter optimization.

The last problem is the change of the number of steady states during the optimization process. We have discussed the importance of three steady states in parameter optimization. However, there is a chance that the system may fail to obtain at least three steady states during optimization process. To solve this problem, we included a steady-state checking step during each run of parameter updating. Specifically, during each run of optimization, we only update one parameter $s_{i j}$ (chosen randomly) instead of all 71 parameters. If that leads to a failure in getting at least three steady states, another parameter $s_{i j}$ will be chosen instead and the learning rate will be changed to a smaller value. The optimization procedure is terminated once the phase change (i.e., the number of steady states changes) happens 10 times in a row. The results of loss function for different learning rate are shown in Fig. 9(a). We also performed a test starting from the second best set of parameters from random search to see the validity and robustness of our method, as shown in Fig. 9(b).

We can see from Fig. 9 that the loss decreases during the optimization process, which supports the feasibility of the gradient descent method. The sudden jump in Fig. 9(a) in the curve is a result of phase change between five steady states and four steady states. Also, a smaller learning rate may require longer time in optimization, though not getting an apparent better result. However, the phase change happened much less when the learning rate is relatively smaller, which indicated a more stable optimization. The result in Fig. 9(b) showed that our method was still effective in terms of decreasing the loss when starting from the second best initial parameter values (corresponding to Fig. 2, model 2).

It is worth noting that the relative loss failed to reach 0 before the optimization ends. Considering the high dimensionality of the HSC network system, it is acceptable since how to obtain the global optimized parameters for such a complex system remains a challenging job. Our aim is to develop a potential approach for parameter inference that can fit static experimental data. In this way, we can combine the gene network modeling and quantitative expression data, and build more realistic models.
[1] S. H. Orkin and L. I. Zon, Hematopoiesis: An evolving paradigm for stem cell biology, Cell 132, 631 (2008).

[2] H. Cheng, Z. Zheng, and T. Cheng, New paradigms on hematopoietic stem cell differentiation, Protein \& cell 11, 34 (2020).

[3] J. L. Galloway and L. I. Zon, Ontogeny of hematopoiesis: Examining the emergence of hematopoietic cells in the vertebrate embryo, Curr. Top. Dev. Biol. 53, 139 (2003).

[4] A. Di Tullio, T. P. V. Manh, A. Schubert, G. Castellano, R. Månsson, and T. Graf, Ccaat/enhancer binding protein $\alpha$ (c/ebp $\alpha)$-induced transdifferentiation of pre-b cells into macrophages involves no overt retrodifferentiation, Proc. Natl. Acad. Sci. USA 108, 17016 (2011).

[5] Y. Zhang, S. Gao, J. Xia, and F. Liu, Hematopoietic hierarchyAn updated roadmap, Trends Cell Biol. 28, 976 (2018).

[6] L. Yang, D. Bryder, J. Adolfsson, J. Nygren, R. MaŁnsson, M. Sigvardsson, and S. E. W. Jacobsen, Identification of lin-sca1+ kit+ cd34+ flt3-short-term hematopoietic stem cells capable of rapidly reconstituting and rescuing myeloablated transplant recipients, Blood 105, 2717 (2005).

[7] A. Garg, K. Mohanram, G. De Micheli, and I. Xenarios, Implicit methods for qualitative modeling of gene regulatory networks, Gene Regulatory Networks (Springer, Berlin, 2012), pp. 397443.

[8] S. Collombet, C. van Oevelen, J. L. S. Ortega, W. Abou-Jaoudé, B. Di Stefano, M. Thomas-Chollier, T. Graf, and D. Thieffry,
Logical modeling of lymphoid and myeloid cell specification and transdifferentiation, Proc. Natl. Acad. Sci. USA 114, 5792 (2017).

[9] N. Bonzanni, A. Garg, K. A. Feenstra, J. Schütte, S. Kinston, D. Miranda-Saavedra, J. Heringa, I. Xenarios, and B. Göttgens, Hard-wired heterogeneity in blood stem cells revealed using a dynamic regulatory network model, Bioinformatics 29, i80 (2013).

[10] J. Krumsiek, C. Marr, T. Schroeder, and F. J. Theis, Hierarchical differentiation of myeloid progenitors is encoded in the transcription factor network, PloS one 6, e22649 (2011).

[11] S. Bornholdt, Boolean network models of cellular regulation: prospects and limitations, J. R. Soc., Interface 5, S85 (2008).

[12] M. Acar, J. T. Mettetal, and A. Van Oudenaarden, Stochastic switching as a survival strategy in fluctuating environments, Nat. Genet. 40, 471 (2008).

[13] J. Wang, Landscape and flux theory of nonequilibrium dynamical systems with application to biology, Adv. Phys. 64, 1 (2015).

[14] C. H. Waddington, The Strategy of the Genes, a Discussion of Some Aspects of Theoretical Biology (G. Allen and Unwin, Crows Nest, Australia, 1957).

[15] S. Huang, The molecular and mathematical basis of waddington's epigenetic landscape: A framework for post-darwinian biology? Bioessays 34, 149 (2012). 
[16] J. Wang, K. Zhang, L. Xu, and E. K. Wang, Quantifying the Waddington landscape and biological paths for development and differentiation, Proc. Natl. Acad. Sci. USA 108, 8257 (2011).

[17] C. Lv, X. Li, F. Li, and T. Li, Energy landscape reveals that the budding yeast cell cycle is a robust and adaptive multistage process, PLoS Comput. Biol. 11, e1004156 (2015).

[18] C. Li and J. Wang, Landscape and flux reveal a new global view and physical quantification of mammalian cell cycle, Proc. Natl. Acad. Sci. USA 111, 14130 (2014).

[19] M. Lu, J. Onuchic, and E. Ben-Jacob, Construction of an Effective Landscape for Multistate Genetic Switches, Phys. Rev. Lett. 113, 078102 (2014).

[20] H. Ge and H. Qian, Landscapes of nongradient dynamics without detailed balance: Stable limit cycles and multiple attractors, Chaos 22, 023140 (2012).

[21] H. Su, G. Wang, R. Yuan, J. Wang, Y. Tang, P. Ao, and X. Zhu, Decoding early myelopoiesis from dynamics of core endogenous network, Sci. China Life Sci. 60, 627 (2017).

[22] C. Sima, J. Hua, and S. Jung, Inference of gene regulatory networks using time-series data: A survey, Curr. Genomics 10, 416 (2009).

[23] H. C. Nguyen, R. Zecchina, and J. Berg, Inverse statistical problems: From the inverse Ising problem to data science, Adv. Phys. 66, 197 (2017).

[24] C. Spieth, F. Streichert, N. Speer, and A. Zell, Optimizing topology and parameters of gene regulatory network models from time-series experiments, Genetic and Evolutionary Computation Conference (Springer, Berlin, 2004), pp. 461-470.

[25] W. Zhao, E. Serpedin, and E. R. Dougherty, Inferring gene regulatory networks from time series data using the minimum description length principle, Bioinformatics 22, 2129 (2006).

[26] P. Baldi, Gradient descent learning algorithm overview: A general dynamical systems perspective, IEEE Trans. Neural Netw. 6, 182 (1995).

[27] J. Guo and J. Zheng, Hopland: Single-cell pseudotime recovery using continuous hopfield network-based modeling of waddington's epigenetic landscape, Bioinformatics 33, i102 (2017).

[28] C. Li and J. Wang, Quantifying cell fate decisions for differentiation and reprogramming of a human stem cell network: Landscape and biological paths, PLoS Comput. Biol. 9, e1003165 (2013).

[29] B. Zhang and P. G. Wolynes, Stem cell differentiation as a many-body problem, Proc. Natl. Acad. Sci. USA 111, 10185 (2014).

[30] J. Schütte, H. Wang, S. Antoniou, A. Jarratt, N. K. Wilson, J. Riepsaame, F. J. Calero-Nieto, V. Moignard, S. Basilico, S. J. Kinston et al., An experimentally validated network of nine haematopoietic transcription factors reveals mechanisms of cell state stability, Elife 5, e11469 (2016).

[31] A. V. Hill, The possible effects of the aggregation of the molecules of haemoglobin on its dissociation curves, J. Physiol. 40, iv-vii (1910).

[32] J. N. Weiss, The hill equation revisited: Uses and misuses, FASEB J. 11, 835 (1997).

[33] S. Huang, Y. Guo, G. May, and T. Enver, Bifurcation dynamics of cell fate decision in bipotent progenitor cells, Dev. Biol. 305, 695 (2007).
[34] B. Huang, M. Lu, D. Jia, E. Ben-Jacob, H. Levine, and J. N. Onuchic, Interrogating the topological robustness of gene regulatory circuits by randomization, PLoS Comput. Biol. 13, e1005456 (2017).

[35] B. Huang, Y. Xia, F. Liu, and W. Wang, Realization of tristability in a multiplicatively coupled dual-loop genetic network, Sci. Rep. 6, 28096 (2016).

[36] W. R. Holmes, N. S. R. de Mochel, Q. Wang, H. Du, T. Peng, M. Chiang, O. Cinquin, K. Cho, and Q. Nie, Gene expression noise enhances robust organization of the early mammalian blastocyst, PLoS Comput. Biol. 13, e1005320 (2017).

[37] X. Zhang, F. Liu, and W. Wang, Two-phase dynamics of p53 in the dna damage response, Proc. Natl. Acad. Sci. USA 108, 8990 (2011).

[38] Y. Ye, X. Kang, J. Bailey, C. Li, and T. Hong, An enriched network motif family regulates multistep cell fate transitions with restricted reversibility, PLoS Comput. Biol. 15, e1006855 (2019).

[39] M. Lu, M. K. Jolly, H. Levine, J. N. Onuchic, and E. Ben-Jacob, Microrna-based regulation of epithelial-hybrid-mesenchymal fate determination, Proc. Natl. Acad. Sci. USA 110, 18144 (2013).

[40] M. Sasai and P. Wolynes, Stochastic gene expression as a manybody problem, Proc. Natl. Acad. Sci. USA 100, 2374 (2003).

[41] M. Freidlin and M. Weber, Random perturbations of dynamical systems and diffusion processes with conservation laws, Probab. Theory Relat. Fields 128, 441 (2004).

[42] X. Zhou, W. Ren, and W. E, Adaptive minimum action method for the study of rare events, J. Chem. Phys. 128, 104111 (2008).

[43] D. K. Wells, W. L. Kath, and A. E. Motter, Control of Stochastic and Induced Switching in Biophysical Networks, Phys. Rev. X 5, 031036 (2015).

[44] C. Li and J. Wang, Quantifying waddington landscapes and paths of nonadiabatic cell fate decisions for differentiation, reprogramming and transdifferentiation, J. R. Soc. Interface 10, 20130787 (2013).

[45] P. Laslo, C. J. Spooner, A. Warmflash, D. W. Lancki, H. Lee, R. Sciammas, B. N. Gantner, A. R. Dinner, and H. Singh, Multilineage transcriptional priming and determination of alternate hematopoietic cell fates, Cell 126, 755 (2006).

[46] S. Stehling-Sun, J. Dade, S. L. Nutt, R. P. DeKoter, and F. D. Camargo, Regulation of lymphoid versus myeloid fate "choice" by the transcription factor mef2c, Nat. Immunol. 10, 289 (2009).

[47] P. S. Swain, M. B. Elowitz, and E. D. Siggia, Intrinsic and extrinsic contributions to stochasticity in gene expression, Proc. Natl. Acad. Sci. USA 99, 12795 (2002).

[48] M. Kaern, T. C. Elston, W. J. Blake, and J. J. Collins, Stochasticity in gene expression: From theories to phenotypes, Nat. Rev. Genet. 6, 451 (2005).

[49] M. Thattai and O. A. Van, Intrinsic noise in gene regulatory networks, Proc. Natl. Acad. Sci. USA 98, 8614 (2001).

[50] S. L. Nutt, B. Heavey, A. G. Rolink, and M. Busslinger, Commitment to the b-lymphoid lineage depends on the transcription factor pax5, Nature (London) 401, 556 (1999).

[51] M. Sugai, H. Gonda, Y. Nambu, Y. Yokota, and A. Shimizu, Role of id proteins in b-lymphocyte activation: New insights from knockout mouse studies, J. Mol. Med. 82, 592 (2004).

[52] S. R. McKercher, B. E. Torbett, K. L. Anderson, G. W. Henkel, D. J. Vestal, H. Baribault, M. Klemsz, A. J. Feeney, G. E. Wu, 
C. J. Paige et al., Targeted disruption of the pu. 1 gene results in multiple hematopoietic abnormalities, EMBO J. 15, 5647 (1996).

[53] M. A. Zarnegar and E. V. Rothenberg, Ikaros represses and activates pu. 1 cell-type-specifically through the multifunctional sfpi1 ure and a myeloid specific enhancer, Oncogene 31, 4647 (2012).

[54] C. Li, Identifying the optimal anticancer targets from the landscape of a cancer-immunity interaction network, Phys. Chem. Chem. Phys. 19, 7642 (2017).

[55] S. V. Puram, I. Tirosh, A. S. Parikh, A. P. Patel, K. Yizhak, S. Gillespie, C. Rodman, C. L. Luo, E. A. Mroz, K. S. Emerick et al., Single-cell transcriptomic analysis of primary and metastatic tumor ecosystems in head and neck cancer, Cell 171, 1611 (2017).

[56] C. Li and G. Balazsi, A landscape view on the interplay between emt and cancer metastasis, NPJ systems biology and applications 4, 1 (2018).
[57] G. P. Dunn, A. T. Bruce, H. Ikeda, L. J. Old, and R. D. Schreiber, Cancer immunoediting: From immunosurveillance to tumor escape, Nat. Immunol. 3, 991 (2002).

[58] C. Weinreb, S. Wolock, B. K. Tusi, M. Socolovsky, and A. M. Klein, Fundamental limits on dynamic inference from single-cell snapshots, Proc. Natl. Acad. Sci. USA 115, E2467 (2018).

[59] J. Wang, L. Xu, and E. Wang, Potential landscape and flux framework of nonequilibrium networks: Robustness, dissipation, and coherence of biochemical oscillations, Proc. Natl. Acad. Sci. 105, 12271 (2008).

[60] N. G. Van Kampen, Stochastic Processes in Chemistry and Physics, 3rd ed. (North Holland, Amsterdam, 2007).

[61] G. Hu, Stochastic Forces and Nonlinear Systems (Shanghai Scientific and Technological Education Publishing House, Shanghai, 1994).

[62] https://github.com/chunhelilab/HSC. 